\title{
Evaluation of the Management of Urolithiasis with Clinical Study
}

\author{
Mukesh Chande ${ }^{\circledR 1}$, Jaydeep M Gadhavi ${ }^{2}$ \\ ${ }^{1}$ Assistant Professor, Department of Surgery, Gujarat Adani Institute of Medical Science, Bhuj, Kutch, Gujarat, India, ${ }^{2}$ Associate Professor, Department of Surgery, \\ GMERS Medical College, Gandhinagar, Gujarat, India.
}

\section{Abstract}

Background: Urolithiasis encompasses both renal and ureteric stones. It is estimated that up to $5 \%$ of the world population is affected by this disease and the lifetime risk of getting urinary stone is $8-15 \%$. The aim of this study is to look for epidemiological patterns in the disease distribution, understand the causative factors of the disease and assess the existing surgical modalities of treatment of this disease. Subjects and Methods: In the present study 68 cases of Urolithiasis fulfilling the study parameters were admitted and subsequently operated in Medical College and Research Hospital. Results: $65 \%$ of the patients were between age group 18-40. The most common site of urinary calculus was lower $1 / 3$ ureter followed by lower pole kidney. Diet, fluid intake, diabetes and obesity were significant predisposing factors for urolithiasis. PCNL was the treatment of choice for renal calculus while ECSWL was treatment of choice in upper ureteric calculi and ureteroscopy for lower ureteric calculi. Conclusion: For renal calculi, PCNL is the best treatment modality as of now, but it is associated with greater post-operative morbidity. For stone less than $1 \mathrm{~cm}$ size, ECSWL is a good alternative to PCNL, but has poor clearance rate and thus greater need for auxiliary procedure. For ureteric calculi, both ECSWL and ureteroscopy have given good results but ECSWL is better tolerated by the patients.

Keywords: Calculi, Urolithiasis, Management, Symptoms

Corresponding Author: Jaydeep M Gadhavi, Associate Professor, Department of Surgery, GMERS Medical College, Gandhinagar, Gujarat, India.

E-mail: drjmg75@gmail.com

Received: 11 April 2020

Revised: 23 May 2020

Accepted: 28 May 2020

Published: 27 May 2020

\section{Introduction}

Urolithiasis refers to a condition characterized by the formation or occurrence of calculi in the urinary tract. ${ }^{[1]}$ Urolithiasis is the third most common urinary tract disease in humans, following urinary tract infections and prostatic diseases. ${ }^{[1]}$ According to localization, the stone maybe present in one or more sites i.e. in the kidney, ureter, bladder and urethra. Urolithiasis refers to a condition characterized by the formation or occurrence of calculi in the urinary tract. ${ }^{[2,3]}$ Urolithiasis is the third most common urinary tract disease in humans, following urinary tract infections and prostatic diseases. According to localization, the stone maybe present in one or more sites i.e. in the kidney, ureter, bladder and urethra. ${ }^{[2]}$

Urolithiasis is a common disease, estimated to affect $11 \%$ of men and $7 \%$ of women in their lifetime. Ureteral stones can cause acute unilateral flank pain radiating to the groin, often accompanied by nausea, vomiting, and urinary symptoms. ${ }^{[4]}$ More than 1 million patients with suspected Urolithiasis present to an emergency department each year in the United
States. ${ }^{[5]}$

Males are more commonly afflicted than females. Increased testosterone levels in men causing increased endogenous oxalate production by the liver and protective increased urinary citrate concentrations in women have been postulated as causes for the same. ${ }^{[6]}$ Various studies over time have shown the cause of urinary stones to be multi-factorial. Urolithiasis commonly presents as pain abdomen. In renal stones, fixed renal pain is common. In the ureteric stones: If in the upper one-third of the ureter - pain radiates to the perineum, if at the pelvic brim - pain radiates to the inner aspect of the thigh, if present in the middle one-third of ureter - pain radiates to the iliac fossa. ${ }^{[7]}$ If the stone is localized in the bladder neck or urethra - pain may present as tip of penis pain. Besides pain, vomiting, nausea, fever, increased frequency of urination, oliguria, dribbling of urine, and hematuria may be seen. ${ }^{[8]}$

The surgical management of stones in patients after urinary diversion is challenging. Open operation monotherapy has a limited role in the treatment of Urolithiasis in these patients on account of the high recurrence rate of stones, postopera- 
tive scar, tissue adhesion and the changed anatomy. ${ }^{[9]}$ The advancement in equipments and increasing experience are making minimally invasive endourologic techniques an appropriate alternative choice for these cases as seen in several reports. ${ }^{[10]}$ However, urinary stone disease is notorious for high recurrence rates even with modern medicine and surgery. Hence, it becomes imperative to study in great details about this disease. Hence the aim of the study was to assess the different modalities of treatment so that an appropriate treatment regimen can be instituted for the patient and further complications may be prevented.

\section{Subjects and Methods}

All the included patients were admitted in the surgical wards of the medical college and hospital. A total of 68 cases were included in the study. Detailed history was taken and the thorough physical examination as per a pre-prepared proforma. Complete data was collected retrospectively, written informed consents were obtained from all participants, and the present study was approved by the Ethics Committee of the First Affiliated Hospital. The routine urine, blood investigations, ultrasonogrphy, abdominal plain X-ray film of kidney, ureter, and bladder (KUB) was done. All patients received non-contrast helical CT scan to evaluate the stone characteristics and peripheral organ disposition. Patients with preoperative positive urine culture received a complete course of culture specific antibiotics treatment. Prophylactic antibiotic was administered to all patients before surgery.

The operative finding, intra- and post-operative complications were recorded. Stones were analyzed using infrared spectroscopy to identify the stone composition. KUB and CT scan was performed to evaluate the stone free status. The success was defined as complete clear or the presence of stone fragment less than $4 \mathrm{~mm}$ without any clinical symptoms.

Depending on the size and site of the calculus, the appropriate treatment for the patient will be decided. The treatment includes Extracorporeal Shock Wave Lithotripsy (ECSWL), Percutaneous nephrolithotomy (PCNL), therapeutic ureteroscopy and Cystoscopic removal of bladder calculi.

\section{Results}

In the present study the patients diagnosed with Urolithiasis and those who fulfilled the criteria of the research were included in the study. In the present study, total of 68 cases were included in the study. Males were more affected as compared to females. There were 27 males and 39 females. Maximum cases were from age 20 - 30 years.

Pain Abdomen was the most common symptom, presenting in 61 patients. This was followed by vomiting/ nausea in 40

\begin{tabular}{|ll|}
\hline Table 1: Distribution of study cases according to age \\
\hline Age group & No. of patients \\
\hline $20-30$ & 27 \\
$31-40$ & 17 \\
$41-50$ & 10 \\
$51-60$ & 14 \\
\hline
\end{tabular}

patients, burning micturation in 33 patients, fever in 14 patients and haematuria in 2 patients. Vomiting, burning micturation and fever was more common in patients of older age group and this was statistically significant $(\mathrm{p}<0.01)$.

\begin{tabular}{ll}
\hline Table 2: Clinical symptoms seen in the patients \\
\hline Clinical symptoms & No. of patients \\
\hline Pain abdomen & 61 \\
Vomiting/Nausea & 40 \\
Burning micturation & 33 \\
Fever & 14 \\
Haematuria & 2 \\
\hline
\end{tabular}

The lower $1 / 3$ of ureter/ vesico-ureteric junction was the most common site of calculus affecting in 26 cases. Lower pole of kidney was the second most common site, seen in 17 cases. Upper 1/3 ureter 8, Middle 1/3 ureter 7 and upper pole kidney 6 followed in frequency. 2 cases had vesical calculus and 1 case had calculus impacted in penile part of the urethra. Male: female ratio for renal stone was 1.13: 1, while it was 2.4:1 in case of ureteric stones. Ureteroscopy was done in 35 cases, PCNL in 22 cases and ECSWL in 7 cases. 3 vesical stores were extracted by open cystolithotomy. Overall PCNL and URS had higher success rates as compared to ECSWL.

\section{Discussion}

Although urinary stone disease is one of the most common afflictions of modern society, it has been described since antiquity. With Westernization of global culture, however, the site of stone formation has migrated from the lower to the upper urinary tract and the disease once limited to men is increasingly gender blind. ${ }^{[11]}$ Until recently, urinary stones in developing countries were considered to be very different from those observed in industrialized countries. Over the last few decades, lifestyle and dietary habits have been westernized in India. Subsequently, the age and sex distribution, etiological factors and management of this disease has seen a major change. [12]

About 30-40 years ago, the male: female ratio was approximately $6: 1-8: 1$. But over a period of time this ratio has decreased to 2:1 worldwide. This is even lower in western 
countries. It has been theorized that the relative increase in number of females inflicted with urolithiasis in western countries is due to modern day dietary habits and lifestyle. In the present study the male: female patient ratio was approximately 2:1, similar to global trends.

Several authors have demonstrated that urolithiasis usually occurs between the third and fourth decades of an individual's life, and that the prevalence rate varies considerably according to age, while the peak incidence of urinary calculi is from the twenties to the forties. Our study showed similar results with nearly $65 \%$ of the study patients between age $20-40$ yrs. It is a matter of concern that the age of presentation of urolithiasis has gradually decreased over the past few decades. Whereas the mean age for urolithiasis was 46.1 in a study done by Hiatt et al the mean age in our study was 32.3.

The goal of the surgical treatment of patients suffering from ureteric calculi is to achieve complete stone clearance with minimal attendant morbidity. In a study by Segura et al, ${ }^{[13]}$ the stone free rates of ECSWL and ureteroscopy were $84 \%$ and $56 \%$, respectively, for stones smaller than $1 \mathrm{~cm}$ and $72 \%$ and $44 \%$, respectively, for stones larger than $1 \mathrm{~cm}$. The risks of significant complications after ECSWL and ureteroscopy were $4 \%$ and $11 \%$, respectively. The present study differed in this respect. Among our patients of ureteric calculus, the success rate was $84.5 \%$ with ureteroscopy compared to $77 \%$ with ECSWL. The post operative complications, though, were higher with ureteroscopy in line with the world literature.

\section{Conclusion}

For renal calculi, PCNL is the best treatment modality as of now, but it is associated with greater post-operative morbidity. For stone less than $1 \mathrm{~cm}$ size, ECSWL is a good alternative to PCNL, but has poor clearance rate and thus greater need for auxiliary procedure. For ureteric calculi, both ECSWL and ureteroscopy have given good results but ECSWL is better tolerated by the patients.

\section{References}

1. Nicolle LE. A practical guide to antimicrobial management of complicated urinary tract infection. Drugs Aging. 2001;18:243-54.

2. Prstojevic J, Junuzovic D, Hasanbegovic M, Lepara Z, Selimovic M. Characteristics of Calculi in the Urinary Tract. Materia Socio Medica. 2014;26:297-297. Available from: https://dx.doi.org/10.5455/msm.2014.26.297-302. doi:10.5455/msm.2014.26.297-302.
3. Junuzovic D, Prstojevic J, Hasanbegovic M, Lepara Z. Evaluation of Extracorporeal Shock Wave Lithotripsy (ESWL): Efficacy in Treatment of Urinary System Stones. Acta Informatica Medica. 2014;22(5):309-309. Available from: https://dx.doi.org/10.5455/aim.2014.22.309-314. doi:10.5455/aim.2014.22.309-314.

4. Wang RC. Managing urolithiasis. Ann Emerg Med. 2016;67:449-54.

5. Fwu CW, Eggers PW, Kimmel PL, Kusek JW, Kirkali Z. Emergency department visits, use of imaging, and drugs for urolithiasis have increased in the United States. Kidney Int. 2013;83(3):479-486. Available from: https://dx.doi.org/10. 1038/ki.2012.419. doi:10.1038/ki.2012.419.

6. Arya MC, Hariyawat BS, Vasudeo V, Jadaon AS, Gandhi A, Singhal A. PCNL in Staghorn Calculi: Our Experience. Asian J Med Res. 2019;8(3):SG01-SG03.

7. Sabharwal S, and NJ. Efficacy of Alpha-1 Adrenergic Blockers in the Management of Benign Prostatic Hyperplasia. Asian Journal of Medical Research. 2019;8(2):SG01SG05. Available from: https://dx.doi.org/10.21276/ajmr.2019. 8.2.sg1. doi:10.21276/ajmr.2019.8.2.sg1.

8. Helfand B, Mouli S, Dedhia R, McVary KT. Management of Lower Urinary Tract Symptoms Secondary to Benign Prostatic Hyperplasia With Open Prostatectomy: Results of a Contemporary Series. J Urol. 2006;176(6):2557-2561. Available from: https://dx.doi.org/10.1016/j.juro.2006.07.143. doi:10.1016/j.juro.2006.07.143.

9. Stuurman RE, Al-Qahtani SM, Cornu JN, Traxer O. Antegrade Percutaneous Flexible Endoscopic Approach for the Management of Urinary Diversion-Associated Complications. J Endourol. 2013;27(11):1330-1334. Available from: https://dx. doi.org/10.1089/end.2012.0371. doi:10.1089/end.2012.0371.

10. Dodd GD, Soulen MC, Kane RA, Livraghi T, Lees WR, Yamashita Y, et al. Minimally Invasive Treatment of Malignant Hepatic Tumors: At the Threshold of a Major Breakthrough. Radiographics. 2000;20(1):9-27. Available from: https://dx.doi.org/10.1148/radiographics.20.1.g00ja019. doi:10.1148/radiographics.20.1.g00ja019.

11. Ahangar S, Durrani AM, Qadri SJ, Patloo AM, Ganaie RG, Khan M. Laparoscopic trans-peritoneal pyelolithotomy in a pelvic kidney. Saudi J Kidney Dis Transplant. 2012;23:1254 58.

12. López M, Hoppe B. History, epidemiology and regional diversities of urolithiasis. Pediatr Nephrol. 2010;25(1):49-59. Available from: https://dx.doi.org/10.1007/s00467-008-09605. doi:10.1007/s00467-008-0960-5.

13. Segura JW, Preminger GM, Assimos DG, Dretler SP, Kahn RI, Lingeman JE, et al. Ureteral Stones Clinical Guidelines Panel Summary Report on the Management of Ureteral Calculi. J Urol. 1997;158(5):1915-1921. Available from: https://dx. doi.org/10.1016/s0022-5347(01)64173-9. doi:10.1016/s00225347(01)64173-9. 
Copyright: (C) the author(s), 2020. It is an open-access article distributed under the terms of the Creative Commons Attribution License (CC BY 4.0), which permits authors to retain ownership of the copyright for their content, and allow anyone to download, reuse, reprint, modify, distribute and/or copy the content as long as the original authors and source are cited.

How to cite this article: Chande M, Gadhavi JM. Evaluation of the Management of Urolithiasis with Clinical Study. Acad. J Surg. 2020;3(1):107-110.

DOI: dx.doi.org/10.47008/ajs/2020.3.1.23

Source of Support: Nil, Conflict of Interest: None declared. 